\title{
A pair of isoleucyl-tRNA synthetases in Bacilli fulfill complementary roles to enhance fitness and provide antibiotic resistance
}

\author{
Vladimir Zanki ${ }^{1}$, Bartol Bozic ${ }^{1}$ \#, Marko Mocibob $^{1}$, Nenad Ban $^{2}$, Ita Gruic-Sovulj $^{1 *}$
}

${ }^{1}$ Department of Chemistry, Faculty of Science, University of Zagreb, Horvatovac 102a, 10 000 Zagreb, Croatia

2 Department of Biology, Institute of Molecular Biology and Biophysics, ETH Zurich, OttoStern-Weg 5, CH-8093 Zurich, Switzerland.

* Corresponding author: Tel: +3851 4606230, E-mail: gruic@chem.pmf.hr

\# Present address: Drug metabolism and pharmacokinetics, Fidelta Ltd., Hondlova 2/6, 10 000 Zagreb, Croatia

\begin{abstract}
Isoleucyl-tRNA synthetase (IleRS) is an essential enzyme that covalently couples isoleucine to the corresponding tRNA. Bacterial IleRSs group in two clades, ileS1 and ileS2, the latter bringing resistance to the natural antibiotic mupirocin. Generally, bacteria rely on either ileS1 or ileS2 as a standalone housekeeping gene. However, we have found an exception by noticing that Bacillus species with genomic ileS2 consistently also keep ileS1, which appears mandatory in the genus Bacillus. Taking Bacillus megaterium as a model organism, we showed that BmIleRS1 is constitutively expressed, while BmIleRS2 is stress-induced. Both enzymes share the same level of the aminoacylation accuracy. Yet, BmIleRS1 exhibited a two-fold faster aminoacylation turnover $\left(k_{\mathrm{cat}}\right)$ than BmIleRS2 and permitted a notably faster cell-free translation. At the same time, BmIleRS2 displayed a $10^{4}$-fold increase in its $K_{\mathrm{i}}$ for mupirocin, arguing that the aminoacylation turnover in IleRS2 could have been traded-off for antibiotic resistance. As expected, a $B$. megaterium strain deleted for ileS2 was mupirocin-sensitive. We also succeed in the construction of a mupirocin-resistant strain lacking ileS1, a solution not found among Bacilli in nature. However, B. megaterium $\Delta i l e S 1$ displayed a severe fitness loss and was seriously compromised in biofilm formation. Neither of these phenotypes were rescued by BmIleRS2 expressed from the constitutive promotor of ileS1. Thus, BmIleRS1 safeguards $B$. megaterium fitness, likely by promoting faster translation than BmIleRS2, whereas the later enzyme is maintained to provide antibiotic resistance when needed. Our data are consistent with an emerging picture in which fast-growing organisms predominantly use IleRS1 for competitive survival.
\end{abstract}




\section{Introduction}

Bacteria respond to various stress conditions by changes in transcription and/or translation [1, 2]. Important players in the expression of genetic information are aminoacyl-tRNA synthetases (aaRSs) [3, 4]. These housekeeping enzymes supply ribosomes with aminoacylated tRNAs (aatRNA) and secure the optimal speed of translation and cellular fitness under normal and starvation conditions [5]. Aminoacylation is a two-step reaction localized within the aaRS synthetic site [3]. The amino acid is firstly activated at the expense of ATP to form an aminoacyl-adenylate intermediate (aa-AMP) followed by transfer of the aminoacyl moiety to the tRNA (Figure 1A). Coupling of non-cognate aa-tRNA pairs can jeopardize translational fidelity, and this potentially damaging scenario is prevented by hydrolysis of misaminoacylated tRNA (post-transfer editing) at a separate aaRS editing domain. Some aaRS may also hydrolyze non-cognate aminoacyl-adenylate (pre-transfer editing) within the synthetic site [6].

Isoleucyl-tRNA synthetase (IleRS) decodes isoleucine codons. Misaminoacylation of the amino acids that strongly mimic isoleucine, such as valine and non-proteinogenic norvaline, is prevented by IleRS pre- and post-transfer editing (Figure 1A) [7, 8]. IleRSs group in two distinct clades that differ in the structure of the anticodon binding domain, unique sequence elements of the catalytic site and susceptibility to the natural antibiotic mupirocin $[8,9]$. The first clade (IleRS1) comprises most of bacterial as well as all eukaryote mitochondrial IleRSs. The members of the second clade (IleRS2), showing a significantly lower susceptibility to competitive inhibition by mupirocin [10], are found in some bacteria and in the eukaryotic cytoplasm. In general, bacteria rely on either ileS1 or ileS2 for housekeeping functionality. Interestingly, the simultaneous presence of both genomic ileSs in the same organism, besides mupirocin-producing P. fluorescens NCIMB 10586 strain [11], appears to be localized to family Bacillaceae [12].

Here, we used comprehensive bioinformatics, kinetics, mass spectrometry, and in vivo analyses to understand the reasons why these organisms maintain two ileS genes. Using Bacillus megaterium as a model organism, we found that each paralogue provides the advantage of a distinct relevance. BmIleRS2 allows growth on mupirocin, while BmIleRS1 enables a competitive and fast growth phenotype and biofilm formation. Our data suggests that Bacilli, as generally fast-growing organisms, could not compromise with the slow catalytic turnover of BmIleRS2. Instead, they keep ileS1, which supports a higher speed of translation, while they benefit from an acquired antibiotic resistance via the second gene. 


\section{Results}

Phylogenetic analysis suggests ileS1 gene is mandatory in Bacilli

Here, we extended our earlier phylogenetic analysis of two bacterial IleRS clades (IleRS1 and IleRS2) [8] to explore a previously noted small bacterial niche within the family Bacillaceae in which organisms carry both ileS genes in the same genome (Figure 2, purple nodes). In depth analysis revealed that Bacilli surprisingly always have ileS1 while several species also have ileS2 (i.e. B. megaterium). The rationale for acquiring ileS2 is clear (lower susceptibility to mupirocin inhibition [8]). However, what is not clear is why ileS2 cannot serve as the sole housekeeping gene in Bacilli as it does in other species like Actinobacteria and Chlamydia (Figure 2, blue nodes). Indeed, a detailed public database search did not reveal a single Bacilli species lacking ileS1. This indicates that IleRS1 is under strong positive selection in Bacilli.

\section{BmIleRS2 binds mupirocin with $10^{4}$-fold lower affinity relative to BmIleRS1}

To address a rationale for the mandatory presence of ileS1, we chose $B$. megaterium as a model organism and its two IleRSs as model enzymes. We started with in vitro characterization of mupirocin inhibition (Figure 1). Analogously to stand-alone IleRS2s [8, 13], BmIleRS2 displayed a significant resistance to the antibiotic with a moderately high $K_{\mathrm{i}}(1 \mu \mathrm{M})$ measured at amino acid activation, the first step of the two-step aminoacylation reaction (Figure 3A). In contrast, BmIleRS1 was readily inhibited by nanomolar mupirocin and featured a more complex character with non-linear time courses characteristic for slow-binding inhibition (Figure 3B left). The $K_{\mathrm{i}}$ obtained for BmIleRS1 (Figure 3B right) is comparable with the $K_{\mathrm{i}}$ for Staphylococcus aureus IleRS1 [14]. Thus, BmIleRS1 and BmIleRS2 display a $10^{4}$-fold difference in sensitivity to mupirocin in accordance with the prediction that BmIleRS2 provides mupirocin resistance to $B$. megaterium.

\section{BmIleRS1 is two-fold faster in Ile-tRNA $A^{I l e}$ synthesis than BmIleRS2}

BmIleRS2 insensitivity to mupirocin could have evolved through a trade-off between enzyme efficiency and/or accuracy. To explore this possibility, we first compared the BmIleRS1 and BmIleRS2 catalytic competences. We found that BmIleRS1 is indeed 10-fold more efficient in isoleucine activation (measured as the $k_{\mathrm{cat}} / K_{\mathrm{M}}$ ) relative to BmIleRS2 (Table 1). Along the same line, BmIleRS1 appeared superior to BmIleRS2 in the two-step aminoacylation by showing a two-fold higher catalytic turnover ( $k_{\text {cat }}$, Table 2, Supplementary Figure S1). However, at the level of $k_{\text {cat }} / K_{\mathrm{M}}$ BmIleRS1 equals BmIleRS2, due to the opposing $K_{\mathrm{M}}$ (Ile) trends in overall aminoacylation (Table 2) and the isolated activation step (Table 1). Although the origin of this effect is not completely clear, an increase in the aminoacylation $K_{\mathrm{M}}$ (Ile) for BmIleRS1 can be attributed to the modulation of Ile recognition by tRNA (Supplementary Table S1) [7, 15]. Nevertheless, given the cellular concentration of Ile $(\sim 300 \mu \mathrm{M})[16]$, BmIleRS1 and BmIleRS2 may operate at near saturating conditions in vivo. Thus, the higher aminoacylation turnover $\left(k_{\text {cat }}\right)$ of BmIleRS1 may be relevant in the cell. 


\section{BmIleRS1 maintains ribosomal translation better than BmIleRS2}

To what extent might a 2-fold difference in aminoacylation turnover influence protein translation? This question was addressed using a cell-free coupled transcription-translation system (PURExpress, NEB) deprived of E. coli IleRS. BmIleRS1 or BmIleRS2 were added at the same concentrations and synthesis of a dihydrofolate reductase (DHFR) reporter enzyme containing 12 Ile residues was monitored by incorporation of $\left[{ }^{35} \mathrm{~S}\right]$-methionine. Under conditions where protein translation was limited by Ile-tRNA ${ }^{\text {Ile }}$ synthesis, BmIleRS1 accumulated more DHFR compared to BmIleRS2, especially at prolonged translation $(>2$ hours, Figure 4). Importantly, under these conditions stability and activity of BmIleRS2 was not affected (Supplementary Figure S2). Thus, the cell-free data endorse in vitro kinetic analysis by showing a noticeable superiority of BmIleRS1 in translation.

\section{Discrimination against noncognate amino acids is conserved between BmIleRSs}

The next question was whether the BmIleRS2 fidelity is compromised due to the increased insensitivity to mupirocin. Therefore, activation of the near-cognate, L-Val and L-Nva, and also D-Ile was tested, and the discrimination factors $(D)$ calculated (Table 1). Low $D$ implies frequent misactivation and, based on the anticipated tolerable translational error [17], $D<3300$ is taken as an indication for editing. The data show that BmIleRS1 and BmIleRS2 discriminate to a similar extent against L-Val and L-Nva, mainly through the $K_{\mathrm{M}}$ effect (Table 1) as other IleRSs [7, 8]. Both enzymes also discriminate similarly against D-Ile, pointing that the existence of the two enzymes is not related to the possible use of D-cognate amino acids in peptide wall synthesis, as in the case of B. subtilis TyrRS [18]. Thus, in contrast to the catalytic turnover which appears to be compromised in BmIleRS2, the enzymes do not differ in initial amino acid selectivity.

\section{Both enzymes feature post-transfer editing and lack pre-transfer editing}

Initial amino acid selectivity and editing jointly modulate IleRS accuracy. So, we next questioned whether BmIleRS1 and BmIleRS2 differ in their post-transfer editing capacity (Figure 1). We prepared Val- and Nva- $\left[{ }^{32} \mathrm{P}\right]-\mathrm{tRNA}{ }^{\text {Ile }}$ and mixed them with an excess of enzyme (see Supplementary Materials and methods) to measure the first-order rate constants describing the hydrolysis of misacylated tRNAs $\left(k_{\text {deacyl }}\right)$. The obtained rates were similar (Supplementary Table S2) demonstrating that BmIleRS1 and BmIleRS2 resemble each other in post-transfer editing. The rates were about $10^{4}$-fold faster than the non-enzymatic hydrolysis (Supplementary Table S2) showing that BmIleRSs, like other IleRSs [7, 8], feature rapid post-transfer editing.

Besides post-transfer editing, error correction at the level of misaminoacyl-AMPs (pretransfer editing, Figure 1) was previously reported for E. coli IleRS [19, 20]. This editing pathway can be separately measured using mutants disabled in post-transfer editing [21]. To explore whether the $B$. megaterium paralogues feature also this editing step, the post-transfer editing inactive variants, BmIleRS1 D333A/T234R and BmIleRS2 D324A/T225R (Supplementary Table S2), were tested in parallel steady-state assays that follow the 
formation of aa-tRNA ${ }^{\text {Ile }}$ ( $k_{\text {aa-tRNA }}$ ) and the consumption of ATP (measured as AMP formation, $k_{\mathrm{AMP}}$ ) [22]. The mutants consumed stoichiometric amount of ATP per synthesized aa-tRNA $\left(k_{\mathrm{AMP}} / k_{\mathrm{aa}-\mathrm{tRNA}}\right.$ ratios for Nva and Val close to 1 ; Supplementary Table S3), demonstrating that both BmIleRS paralogues lack pre-transfer editing. As expected, the WT enzymes exhibit $k_{\mathrm{AMP}} / k_{\text {aa-tRNA }}$ ratios for Val and Nva substantially higher than 1 (Supplementary Table S3) in agreement with strong post-transfer editing. In summary, BmIleRS paralogues exhibit no difference in the accuracy of aminoacylation. The main distinction between the paralogues remains the aminoacylation turnover, which is lower in BmIleRS2 as a possible trade-off with mupirocin resistance.

\section{Expression of BmIleRS2 is highly upregulated by mupirocin}

Does the rationale for BmIleRS1 requirement lie, besides its kinetic superiority, also in its regulation? To address this question, we performed bioinformatic analyses that indicated a housekeeping mode of regulation for ileS1 with its promoter being recognized by RNA polymerase containing $\sigma^{70}$. In contrast, the ileS2 promoter seems to be regulated by stress repressor proteins lexA and $\arg R 2$ (Figure 5A and Supplementary Figure S3A). We also found conserved T-Box riboswitch structural elements preceding both ileS ORFs (Supplementary Figure S3B). This regulation mechanism, in Gram-positive bacteria, senses the aminoacylation state of the tRNA and dictates either read-through or termination of transcription via tRNA:mRNA interactions [23].

The anticipated BmIleRS1 and BmIleRS2 expression profiles were tested by mass spectrometry (MS). Without mupirocin (the control sample), BmIleRS1 was readily detectable, while the BmIleRS2 peptides were close to the detection limit. Addition of mupirocin upregulated BmIleRS2 expression by approximately 70-fold, BmIleRS1 by 2-fold, and did not affect expression of any other aaRS (Figure 5B). SDS-PAGE fractionation followed by in-gel digestion improved sequence coverage and allowed for a better estimate of the relative abundance of BmIleRS1 and BmIleRS2 (Supplementary Figure S4). These data are consistent with BmIleRS1 being constitutively expressed while BmIleRS2 is induced by antibiotic stress to the slightly lower level than BmIleRS1 in the absence of antibiotic.

BmIleRS2 can provide B. megaterium with housekeeping functionality and mupirocin resistance

Our bioinformatic analysis revealed that ileS2 never stands alone in Bacilli. So, a question emerged as to whether BmIleRS2, and specifically under its native inducible promoter, could serve as the sole housekeeping enzyme in Bacilli. To investigate this, we constructed both $B$. megaterium strains deleted for ileS1 or ileS2 (Supplementary Figure S5). The AileS1 strain could be selected only when the cells were grown in presence of mupirocin. However, the constructed strain was viable without mupirocin indicating that expression of BmIleRS2 is constitutive in the absence of ileS1. Importantly, this showed that BmIleRS2, under its native promoter can support housekeeping functionality along with antibiotic resistance (Figure 6A). As expected, the $\Delta i l e S 2$ construction was straightforward and led to mupirocin sensitivity. The 
latter was recovered by expression of BmIleRS2 under its native promoter, from a plasmid (Figure 6B, Supplementary Figure S6A).

\section{BmIleRS1 is superior to BmIleRS2 under nutrient-depleted conditions}

To explore further why BmIleRS2 was not selected during evolution as a stand-alone solution in Bacilli, we performed phenotypic characterization of our knockout strains. In minimal medium, $\Delta i l e S 1$ displayed a 10\% reduced doubling time compared to the WT and $\Delta i l e S 2$ strains (Figure 6). Accordingly, $\triangle i l e S 1$ and $\triangle i l e S 2$ competition in culture revealed that $\Delta i l e S 1$ strain was outcompeted by the $\Delta i l e S 2$ strain (Figure 7B left), providing an explanation for IleRS1 being mandatory in Bacilli. In contrast, in rich medium the strains did not show a difference in doubling times (Figure 6), nor did the $\Delta i l e S 2$ strain outcompete $\Delta i l e S 1$ (Figure 7B, right). However, in rich medium, both the WT and $\Delta i l e S 2$ strains displayed a noticeable lag at $\mathrm{OD}_{600}$ 1.2 , reminiscent of diauxic growth, often considered to provide a competitive advantage [24]: this phenotype was lost in the $\Delta i l e S 1$ strain (Figure 6).

\section{Interplay between BmIleRS1 regulation and activity in biofilm formation}

To screen for additional $\Delta i l e S 1$ phenotypes, we tested the knockout strains for biofilm formation. We found that the $\Delta i l e S 1$ strain displayed a distinct phenotype, dubbed "leaky", which was not observed for WT or $\Delta i l e S 2$ strains (Figure 8A). The WT strain, however, displayed the leaky phenotype in the presence of mupirocin, confirming that the phenotype originates from a loss of the functional BmIleRS1. This strongly suggests that the BmIleRS1 canonical aminoacylation activity is essential for biofilm formation.

To investigate the interplay between BmIleRS enzymatic features and ileS regulation, constructs with swapped promoters and ORFs were prepared (Figure 8B). Interestingly, neither of the $\Delta i l e S 1$ strain phenotypes (leaky biofilm, compromised growth, or lack of so called monoauxic feature) were complemented by BmIleRS2, even under the constitutive promoter of ileS1 (Figure 8C, Supplementary Figure S6B, Supplementary Figure S7). Thus, the compromised kinetic features of BmIleRS2 seem to be responsible for the observed phenotypes. To our surprise, BmIleRS1 under the promoter of ileS2 was not able to rescue AileS1 phenotypes even though we confirmed BmIleRS1 expression (Figure 8C, Supplementary Figure S6B, Supplementary Figure S7) arguing that both BmIleRS1 aminoacylation activity and its regulation are important. 


\section{Discussion}

Simultaneous presence of two paralogues in a single cell can reflect a need for: i) distinct enzymatic features, ii) differential regulation, or iii) both $[18,25,26]$. We investigated two IleRS paralogues from $B$. megaterium, each belonging to a distinct clade of bacterial IleRS proteins (dubbed IleRS1 and IleRS2 [8,9]). BmIleRS2 displayed insensitivity to the antibiotic mupirocin, with the $K_{\mathrm{i}}$ in the micromolar range, whereas BmIleRS1 was sensitive to nanomolar concentrations of the antibiotic (Figure 3). Consequently, BmIleRS2, as it is the case for other characterized IleRS2 enzymes [8, 13, 27], supports antibiotic resistance (Figure 6), a feature that likely influence the horizontal gene transfer events observed in ileS2 [8]. Interestingly, although ileS2 can stand alone in a number of bacteria, in Bacilli, ileS2 is present only as a second gene (Figure 2). This opens the intriguing question of whether IleRS2-only organisms experience fitness trade-offs associated with the acquired mupirocin resistance [28]. We rationalized that either aminoacylation fidelity, known to ensure cell viability [7, 29], or aminoacylation speed could be compromised in IleRS2. Detailed kinetic analysis demonstrated that BmIleRS2 is indistinguishable from BmIleRS1 (and other previously tested IleRS1 and IleRS2 enzymes [7, 8]) in terms of initial substrate selectivity (Table 1) or post-transfer editing (Supplementary Table S2). Interestingly, BmIleRS2, similar to some other IleRS2 proteins [8], lacks tRNA-dependent pre-transfer editing within the synthetic site (Supplementary Table S3). However, we found the same for BmIleRS1 (Supplementary Table S3). Taken together, the evolutionary pressure for IleRS1 in Bacilli is apparently not related to aminoacylation fidelity. However, we discovered that the mupirocin resistant BmIleRS2 has two-fold slower aminoacylation turnover $\left(k_{\mathrm{cat}}\right)$ (Table 2), which translates into a noticeably slower cell-free translation (Figure 4), suggesting that mupirocin-resistance may come on account of the translational rate. Could this govern the universal presence of IleRS1 in Bacilli, and even more broadly? Limited kinetic data obtained here and reported previously on related systems show that IleRS1s are indeed faster in aminoacylation than IleRS2s (Table 2, [8, 30]). Also, we collected data on the minimum doubling times of 207 bacteria from the literature and compared this with IleRS distribution (Figure 9). These data revealed that median of the doubling time of bacteria comprising IleRS1 is at least 2-fold lower (i.e. faster growth) than of bacteria relying exclusively on IleRS2. Thus, it appears that IleRS1 is required by fastergrowing bacteria to maintain fast translation (Supplementary Figure S8).

Examination of a $B$. megaterium strain that lacks BmIleRS1 and relies solely on BmIleRS2 (AileS1, Figure 6) revealed that this strain is notably compromised. Specifically, $\Delta i l e S 1$ could not produce biofilm (Figure 8) and in direct competition was outgrown by the $\triangle i l e S 2$ strain (Figure 7). Two reasons may account for these observations: either IleRS1 is mandatory or ileS2 native regulation compromises fitness. BmIleRS2 expression is regulated by i) a stress sensitive promotor and ii) the T-box mechanism [23] (Figure 5 and Supplementary Figure S3). Both promote ileS2 expression when BmIleRS1 is missing ( $\triangle i l e S 1)$ or is mupirocin-inhibited (WT strain) via non-aminoacylated tRNA ${ }^{\text {Ile }}$ that stimulates the stringent response [31] and prevents ileS2 transcriptional attenuation. However, BmIleRS2 under the constitutive promotor of ileS1 did not rescue the $\Delta i l e S 1$ phenotypes (Figure 8), clearly demonstrating that catalytic properties of BmIleRS1 are important for fitness. 
In summary, in vitro and in vivo analyses jointly provide evidence that IleRS1 in Bacilli and other fast-growing bacteria is maintained due to selective pressure for fast translation. In contrast, slow-growing bacteria may trade-off translational speed for the higher mupirocin resistance and rely on IleRS2. Our data explain the evolutionary advantage of having both IleRS paralogues in Bacilli; IleRS1 ensures competitive fitness and biofilm formation in the absence of antibiotic whereas IleRS2 provides mupirocin resistance. Such enzyme pair combinations and the associated genetic control solutions capable of dealing with the selective pressure of antibiotic presence may be more widespread than currently anticipated. 


\section{Materials and methods}

Bacterial strains, growth conditions and cloning

All cloning was performed in E. coli as described [32] or following manufacturer's protocol. All plasmids, PCR products and strains were verified by sequencing. All plasmids are listed in Supplementary Table S4, primers in Supplementary Table S5, and plasmid construction details in Supplementary Table S6. B. megaterium (DSMZ, strain DSM-32) was grown at 30 ${ }^{\circ} \mathrm{C}$ in LB or minimal medium (A5 medium with $0.5 \% \mathrm{w} / \mathrm{v}$ glucose instead of yeast extract) [33], biofilm formation was assayed in LBGM medium [34] and knockout strains were prepared essentially as described [35]. The growth curves were fitted to logistic [36], biphasic or exponential equation (GraphPad Prism).

The competition experiment was performed by growing strains separately in a liquid medium to the mid-exponential phase followed by dilution of each strain to $\mathrm{OD}_{600} 0.015$ and mixing (total $\mathrm{OD}_{600}$ 0.030). The competing culture was regularly refreshed by mixing the aliquot of the culture with fresh medium (dilution factor $10^{3}-10^{4}$ ) every $10-12 \mathrm{~h}$ for 2 days. Before adding the fresh medium, an aliquot of cells was taken, normalized to $\mathrm{OD}_{600} 1$, serially diluted and plated on LB plates with and without $1 \mu \mathrm{M}$ mupirocin. All experiments were performed at least three times using at least two biological replicates.

\section{Production of IleRS enzymes and $t R N A^{\text {Ile }}$}

B. megaterium enzymes (WT and variants) were cloned (Supplementary Tables S4, S5 and S6) and overexpressed in E. coli at $30{ }^{\circ} \mathrm{C}$ (BmIleRS1) and $15{ }^{\circ} \mathrm{C}$ (BmIleRS2). B. megaterium tRNA $^{\mathrm{Ile}}{ }_{\mathrm{GAU}}$ gene was overexpressed in $E$. coli as described [30]. Protein expression in $B$. megaterium was carried out using $\mathrm{pP}_{\mathrm{T} 7}$ (for BmIleRS1) and $\mathrm{pMGBm} 19$ (for BmIleRS2) plasmids [37].

\section{Kinetic assays}

All assays were performed at $30{ }^{\circ} \mathrm{C}$ essentially as described [8, 30]. Slow, tight binding mupirocin inhibition of BmIleRS1 was assayed as described by Morrison and Walsh [38]. For details see Supplementary Materials and methods.

In vitro coupled transcription-translation was performed at $37^{\circ} \mathrm{C}$ using the PURExpress $\triangle$ IleRS Kit (NEB) following manufacturer's protocol supplemented with $\left[{ }^{35} \mathrm{~S}\right]$-methionine (EasyTag, Perkin Elmer) and $20 \mathrm{U}$ murine RNase inhibitor (NEB). BmIleRS enzymes were present at $4 \mathrm{nM}$. Quantification was performed following manufacturer's protocol.

\section{Bioinformatics and phylogenetic analysis}

Phylogenetic tree was constructed as described [8]. Putative promoter elements were analyzed using BacPP [39], FruitFly [40] and PePPER [41] bioinformatics tools and the putative transcription factor binding sites were determined by BProm [42] using approximately 500 nucleotides upstream of the ileS ORF as a query. 


\section{MS analysis}

For the whole proteome analysis, tryptic digests were prepared as described [43]. The digests were analyzed on nanoLC-MS/MS system (EASY-nLC 1200 coupled to Q Exactive Plus mass spectrometer, ThermoFisher Scientific) equipped with EASY-Spray ${ }^{\mathrm{TM}}$ HPLC C18 Column (2 $\mu \mathrm{m}$ particle size, $75 \mu \mathrm{m} / 250 \mathrm{~mm}$ ). ProteomeDiscoverer 2.4 and MaxQuant 1.6.17 were used for data analysis, protein identification and label-free quantification. All samples were prepared and analyzed in triplicates. 


\section{Author contribution}

Vladimir Zanki: investigation (lead), methodology (lead), validation (lead), visualization (lead), writing - original draft preparation (equal), writing - review \& editing (equal)

Bartol Bozic: investigation (supporting), validation (supporting), writing - review \& editing (supporting)

Marko Mocibob: investigation (supporting), validation (supporting), writing - review \& editing (supporting)

Nenad Ban: funding acquisition (equal), writing - review \& editing (supporting)

Ita Gruic-Sovulj: conceptualization (lead), funding acquisition (equal), project administration (lead), supervision (lead), writing - original draft preparation (equal), writing - review \& editing (equal)

\section{Acknowledgments}

We gratefully acknowledge Jeff Errington, Lianet Noda-Garcia, Aleksandra Marsavelski, Igor Zivkovic, Valentina Evic, Mario Kekez and Alojzije Brkic for fruitful discussions regarding the experiments and critical reading of the manuscript, Igor Zivkovic and Maja Baraci for the technical support with the experiments, and Rebekka Biedendieck for providing us with all the plasmids for B. megaterium. I. G.-S. is grateful to Dan S. Tawfik for his highly positive spirit in science and beyond, and for numerous vivid discussions about the IleRS1 and IleRS2 evolution and function.

\section{Funding}

This work was supported by the Swiss Enlargement Contribution in the framework of the Croatian-Swiss Research Programme, Grant IZHRZ0_180567 and European Regional Development Fund (infrastructural project CIuK, grant number KK.01.1.1.02.0016).

\section{Conflict of interest}

The authors declare no conflicts of interest 


\section{References}

1. Boor KJ (2006) Bacterial Stress Responses: What Doesn't Kill Them Can Make Them Stronger. PLoS One 4(1):e23

2. Starosta AL, Lassak J, Jung K et al. (2014) The bacterial translation stress response. FEMS Microbiol Rev 38(6):1172-1201

3. Perona JJ, Gruic-Sovulj I (2014) Synthetic and editing mechanisms of aminoacyl-tRNA synthetases. Top Curr Chem 344:1-41

4. Tawfik DS, Gruic-Sovulj I (2020) How evolution shapes enzyme selectivity - lessons from aminoacyl-tRNA synthetases and other amino acid utilizing enzymes. FEBS J 287(7):12841305

5. Raina M, Michael I (2014) tRNAs as regulators of biological processes. Front Genet 5:171

6. Gruic-Sovulj I, Rokov-Plavec J, Weygand-Durasevic I (2007) Hydrolysis of non-cognate aminoacyl-adenylates by a class II aminoacyl-tRNA synthetase lacking an editing domain. FEBS Lett 581(26):5110-5114

7. Bilus M, Semanjski M, Mocibob M, et al. (2019) On the Mechanism and Origin of Isoleucyl-tRNA Synthetase Editing against Norvaline. J Mol Biol 431(6):1284-1297

8. Cvetesic N, Dulic M, Bilus M, et al. (2016) Naturally Occurring Isoleucyl-tRNA Synthetase without tRNA-dependent Pre-transfer Editing. J Biol Chem 291(6):8618-8631

9. Brown JR, Zhang J, Hodgson JE (1998) A bacterial antibiotic resistance gene with eukaryotic origins. Curr Biol 8(11):R365-367

10. Fuller A, Mellows G, Woolford M, et al. (1971) Pseudomonic acid: an antibiotic produced by Pseudomonas fluorescens. Nature 234(5329):416-417

11. El-Sayed AK, Hothersall J, Cooper SM, et al. (2003) Characterization of the mupirocin biosynthesis gene cluster from Pseudomonas fluorescens NCIMB 10586. Chem Biol 10(5):419-430

12. Brown JR, Gentry D, Becker JA, et al. (2003) Horizontal transfer of drug-resistant aminoacyl-transfer-RNA synthetases of anthrax and Gram-positive pathogens. EMBO Rep 4(7):692-698

13. Nakama T, Nureki O, Yokoyama S (2001) Structural basis for the recognition of isoleucyl-adenylate and an antibiotic, mupirocin, by Isoleucyl-tRNA synthetase. J Biol Chem 276(50):47387-47393

14. Pope AJ, Moore KJ, McVey M, et al. (1998) Characterization of isoleucyl-tRNA synthetase from Staphylococcus aureus. II. Mechanism of inhibition by reaction intermediate and pseudomonic acid analogues studied using transient and steady-state kinetics. J Biol Chem 273(48):31691-31701

15. Cvetesic N, Bilus M, Gruic-Sovulj I (2015) The tRNA A76 Hydroxyl Groups Control Partitioning of the tRNA-dependent Pre- and Post-transfer Editing Pathways in class I tRNA synthetase. J Biol Chem 290(22):13981-13991 
16. Clark VL, Peterson DE, Bernlohr RW (1972) Changes in Free Amino Acid Production and Intracellular Amino Acid Pools of Bacillus licheniformis as s Function of Culture Age and Growth Media. J Bacteriol 112(2):715-725

17. Loftfield RB, Vanderjagt D (1972) The frequency of errors in protein biosynthesis. Biochem J 128(5):1353-1356

18. Williams-Wagner RN, Grundy FJ, Raina M, et al. (2015) The Bacillus subtilis tyrZ Gene Encodes a Highly Selective Tyrosyl-tRNA Synthetase and Is Regulated by a MarR Regulator and T Box Riboswitch. J Bacteriol 197(9):1624-1631

19. Dulic M, Cvetesic N, Perona JJ, et al (2010) Partitioning of tRNA-dependent Editing between Pre- and Post-transfer Pathways in Class I Aminoacyl-tRNA Synthetases. J Biol Chem 285(31):23799-23809

20. Fersht AR (1977) Editing mechanisms in protein synthesis. Rejection of valine by the isoleucyl-tRNA synthetase. Biochemistry 16(5):1025-1030

21. Cvetesic N, Gruic-Sovulj I (2017) Synthetic and editing reactions of aminoacyl-tRNA synthetases using cognate and non-cognate amino acid substrates. Methods 113:13-26

22. Dulic M, Perona JJ, Gruic-Sovulj I (2014) Determinants for tRNA-dependent pretransfer editing in the synthetic site of isoleucyl-tRNA synthetase. Biochemistry 53(39):6189-6198

23. Henkin TM (2014) The T box riboswitch: a novel regulatory RNA that utilizes tRNA as its ligand. Biochim Biophys Acta 1839(10):959-963

24. Salvy P, Hatzimanikatis V (2021) Emergence of diauxie as an optimal growth strategy under resource allocation constraints in cellular metabolism. Proc Natl Acad Sci U S A 118(8):e2013836118

25. Noda-Garcia L, Romero Romero ML, Longo LM, et al. (2017) Bacilli glutamate dehydrogenases diverged via coevolution of transcription and enzyme regulation. EMBO Rep 18(7):1139-1149

26. Putzer H, Gendron N, Grunberg-Manago M (1992) Co-ordinate expression of the two threonyl-tRNA synthetase genes in Bacillus subtilis: control by transcriptional antitermination involving a conserved regulatory sequence. EMBO J 11(8):3117-3127

27. Yanagisawa T, Kawakami M (2003) How Does Pseudomonas fluorescens Avoid Suicide from Its Antibiotic Pseudomonic Acid?. J Biol Chem 278(28):25887-25894

28. Melnyk AH, Wong A, Kassen R (2015) The fitness costs of antibiotic resistance mutations. Evol Appl 8(3):273-283

29. Kermgard E, Yang Z, Michel A-M, et al. (2017) Quality Control by Isoleucyl-tRNA Synthetase of Bacillus subtilis Is Required for Efficient Sporulation. Sci Rep 7:41764

30. Zivkovic I, Moschner J, Koksch B, et al. (2020) Mechanism of discrimination of isoleucyl-tRNA synthetase against nonproteinogenic $\alpha$-aminobutyrate and its fluorinated analogues. FEBS J 287(4):800-813 
31. Hauryliuk V, Atkinson GC, Murakami KS, et al. (2015) Recent functional insights into the role of (p)ppGpp in bacterial physiology. Nat Rev Microbiol 13(5):298-309

32. Sambrook J, Russell D (2001) Molecular Cloning: A laboratory manual. Cold Spring Harbor Laboratory Press

33. Malten M, Hollmann R, Deckwer W-D, et al. (2005) Production and Secretion of Recombinant Leuconostoc mesenteroides Dextransucrase DsrS in Bacillus megaterium. Biotechnol Bioeng 89(2):206-218

34. Shemesh M, Chai Y (2013) A Combination of Glycerol and Manganese Promotes Biofilm Formation in Bacillus subtilis via Histidine Kinase KinD Signaling. J Bacteriol 195(12):2747-2754

35. Biedendieck R, Borgmeier C, Bunk B, et al. (2011) Systems biology of recombinant protein production using Bacillus megaterium. Methods Enzymol 500:165-195

36. Cvetesic N, Palencia A, Halasz I, et al. (2014) The physiological target for LeuRS translational quality control is norvaline. EMBO J 33(15):1639-1653

37. Gamer M, Frode D, Biedendieck R, et al. (2009) A T7 RNA polymerase-dependent gene expression system for Bacillus megaterium. Appl Microbiol Biotechnol 82(6):1195-1203

38. Morrison J, Walsh C (1988) The behavior and significance of slow-binding enzyme inhibitors. Adv Enzymol Relat Areas Mol Biol 61:201-301

39. de Avila E Silva S, Echeverrigaray S, Gerhardt GJL (2011) BacPP: bacterial promoter prediction--a tool for accurate sigma-factor specific assignment in enterobacteria. $\mathrm{J}$ Theor Biol 187:92-99

40. Reese M (2001) Application of a time-delay neural network to promoter annotation in the Drosophila melanogaster genome. Comput Chem 26(1):51-56

41. de Jong A, Pietersma H, Cordes M, et al. (2012) PePPER: a webserver for prediction of prokaryote promoter elements and regulons. BMC Genomics 13:299

42. Solovyev V, Salamov A, Automatic Annotation of Microbial Genomes and Metagenomic Sequences. In: Li RW (2011) Metagenomics and its Applications in Agriculture. Nova Science Publishers, New York, pp 61-78.

43. Cvetesic N, Semanjski M, Soufi B, et al. (2016) Proteome-wide measurement of noncanonical bacterial mistranslation by quantitative mass spectrometry of protein modifications. Sci Rep 6:28631 
Table 1. Steady state amino acid activation ${ }^{a}$

\begin{tabular}{|c|c|c|c|c|c|}
\hline IleRS & substrate & $k_{\text {cat }} / \mathrm{s}^{-1}$ & $K_{\mathrm{M}} / \mu \mathrm{M}$ & $\begin{array}{l}k_{\text {cat }} / K_{\mathrm{M}} / \\
\left(\mathrm{s}^{-1} \mu \mathrm{M}^{-1}\right)\end{array}$ & $\begin{array}{l}\text { discrimination } \\
\text { factor }^{b}\end{array}$ \\
\hline \multirow{5}{*}{ BmIleRS1 } & L-Ile & \multirow{2}{*}{$30 \pm 2$} & $2.1 \pm 0.3$ & 14.3 & \\
\hline & ATP & & $395 \pm 32$ & 0.076 & \\
\hline & L-Val & $25 \pm 1$ & $277 \pm 33$ & 0.090 & 159 \\
\hline & L-Nva & $27 \pm 2$ & $387 \pm 74$ & 0.069 & 207 \\
\hline & D-Ile ${ }^{c}$ & $30 \pm 3$ & $(3.8 \pm 0.3) \times 10^{3}$ & 0.0079 & 1810 \\
\hline \multirow{5}{*}{ BmIleRS2 } & L-Ile & \multirow{2}{*}{$66 \pm 2$} & $48.6 \pm 1.9$ & 1.4 & \\
\hline & ATP & & $(1.6 \pm 0.1) \times 10^{3}$ & 0.04 & \\
\hline & L-Val & $39 \pm 1$ & $(5.4 \pm 0.4) \times 10^{3}$ & 0.007 & 200 \\
\hline & L-Nva & $33 \pm 1$ & $(7.97 \pm 0.84) \times 10^{3}$ & 0.0041 & 341 \\
\hline & D-Ile ${ }^{c}$ & $1.54 \pm 0.03$ & $(2.3 \pm 0.1) \times 10^{3}$ & $6.7 \times 10^{-4}$ & 2090 \\
\hline
\end{tabular}

${ }^{a}$ measured using ATP/PPi exchange assay with BmIleRSs overexpressed in E. coli

${ }^{\mathrm{b}}$ discrimination factor is calculated as $\left(k_{\mathrm{cat}} / K_{\mathrm{M}}\right)_{\text {cognate amino acid }} /\left(k_{\mathrm{cat}} / K_{\mathrm{M}}\right)_{\text {non-cognate amino acid }}$

${ }^{\mathrm{c}} \mathrm{D}$-Val kinetic parameters could not be determined due to insufficient activity and low D-Val solubility

The values represent the average value $\pm \operatorname{SEM}(n \geq 3)$

Table 2. Steady state two-step aminoacylation ${ }^{a, b}$

\begin{tabular}{|c|c|c|c|c|}
\hline IleRS & substrate & $k_{\text {cat }} / \mathrm{s}^{-1}$ & $K_{\mathrm{M}} / \mu \mathrm{M}$ & $k_{\text {cat }} / K_{\mathrm{M}} /\left(\mathrm{s}^{-1} \mu \mathrm{M}^{-1}\right)$ \\
\hline \multirow{3}{*}{ BmIleRS1 } & L-Ile & \multirow{3}{*}{$1.75 \pm 0.12$} & $43.2 \pm 3.3$ & 0.04 \\
\hline & ATP & & $246.0 \pm 19.8$ & 0.01 \\
\hline & tRNA $^{\text {Ile }}$ & & $2.03 \pm 0.15$ & 0.86 \\
\hline \multirow{3}{*}{ BmIleRS2 } & L-Ile & \multirow{3}{*}{$0.96 \pm 0.03$} & $6.2 \pm 0.8$ & 0.15 \\
\hline & ATP & & $76.4 \pm 1.4$ & 0.01 \\
\hline & tRNA $^{\text {Ile }}$ & & $3.7 \pm 0.3$ & 0.26 \\
\hline
\end{tabular}

${ }^{a}$ measured using BmIleRSs overexpressed in B. megaterium

$\mathrm{b}$ the progress curves displayed biphasic nature. The fast phase was used to determine the kinetic parameters (Supplementary Figure 2).

The values represent the average value $\pm \operatorname{SEM}(n \geq 3)$ 
A

two-step aminoacylation (activation + transfer)
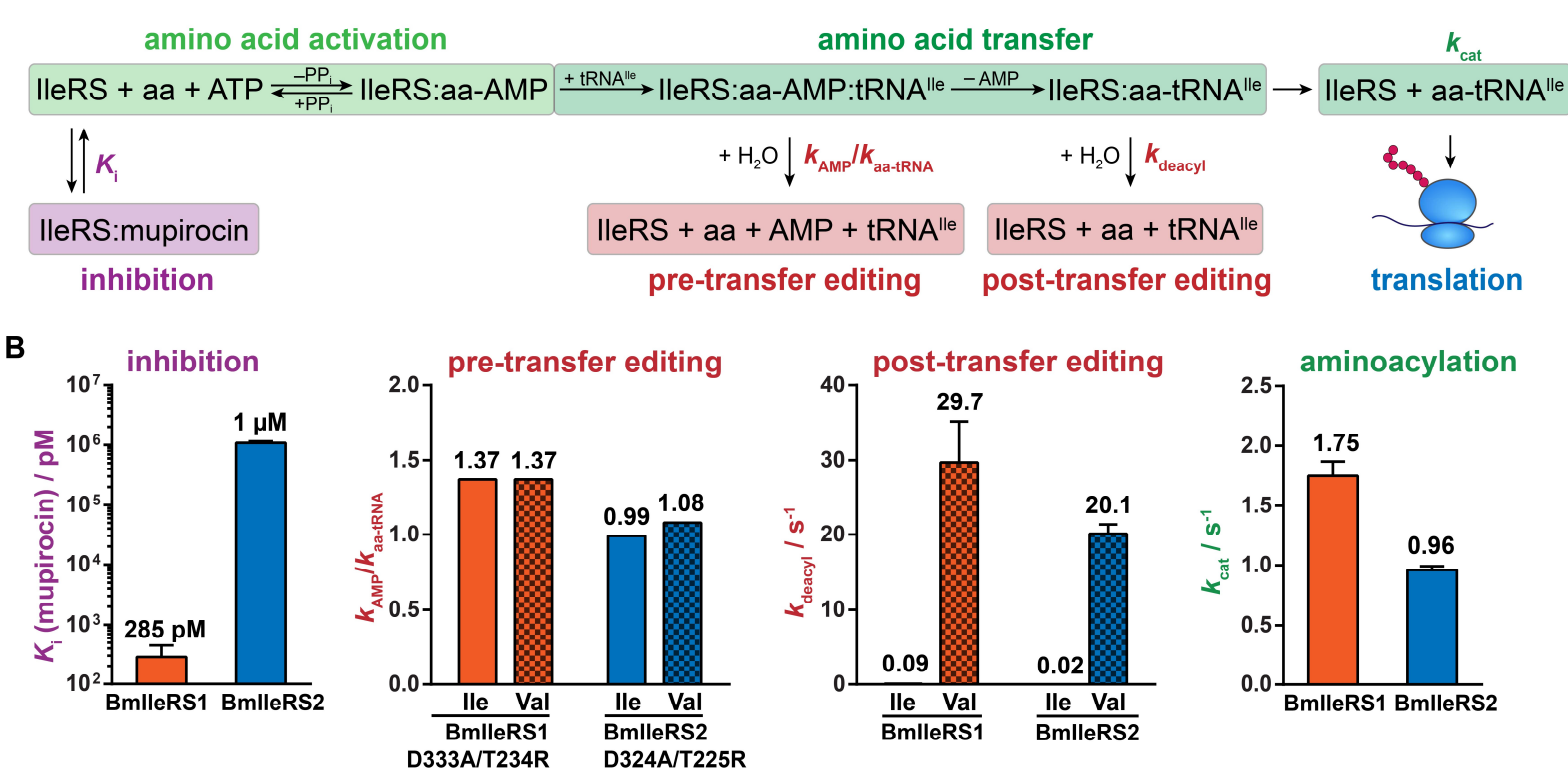

Figure 1. Schematic representation of IleRS aminoacylation and editing reactions with the corresponding key biochemical results.

A) A two-step synthetic pathway (green) leads to synthesis of Ile-tRNA ${ }^{\text {Ile }}$, represented by aminoacylation turnover, $k_{\text {cat }}$. IleRS inhibition by antibiotic mupirocin is described by inhibition constant, $K_{\mathrm{i}}$ (purple). The editing pathways (red) comprise i) tRNA-dependent pretransfer editing, calculated as the ratio of the steady-state rate constants for ATP consumption and aa-tRNA ${ }^{\text {Ile }}$ synthesis in the presence of cognate or non-cognate (Val) amino acid catalyzed by the post-transfer editing-defective IleRS variant (ratio higher than one indicates editing) and ii) post-transfer editing, represented by the single-turnover rate constant for aa-tRNA ${ }^{\text {Ile }}$ hydrolysis, $k_{\text {deacyl }}$.

B) Kinetic data unveil that BmIleRS2, relative to BmIleRS1, has a higher $K_{\mathrm{i}}$ for mupirocin (Figure 3), the same post-transfer editing rate (Supplementary Table S3) but lower aminoacylation $k_{\text {cat }}$ (Table 2). Both BmIleRSs lack pre-transfer editing (Supplementary Table S2). The same editing results were obtained also with non-cognate Nva. Amino acid activation parameters are given in Table $\mathbf{1}$. 


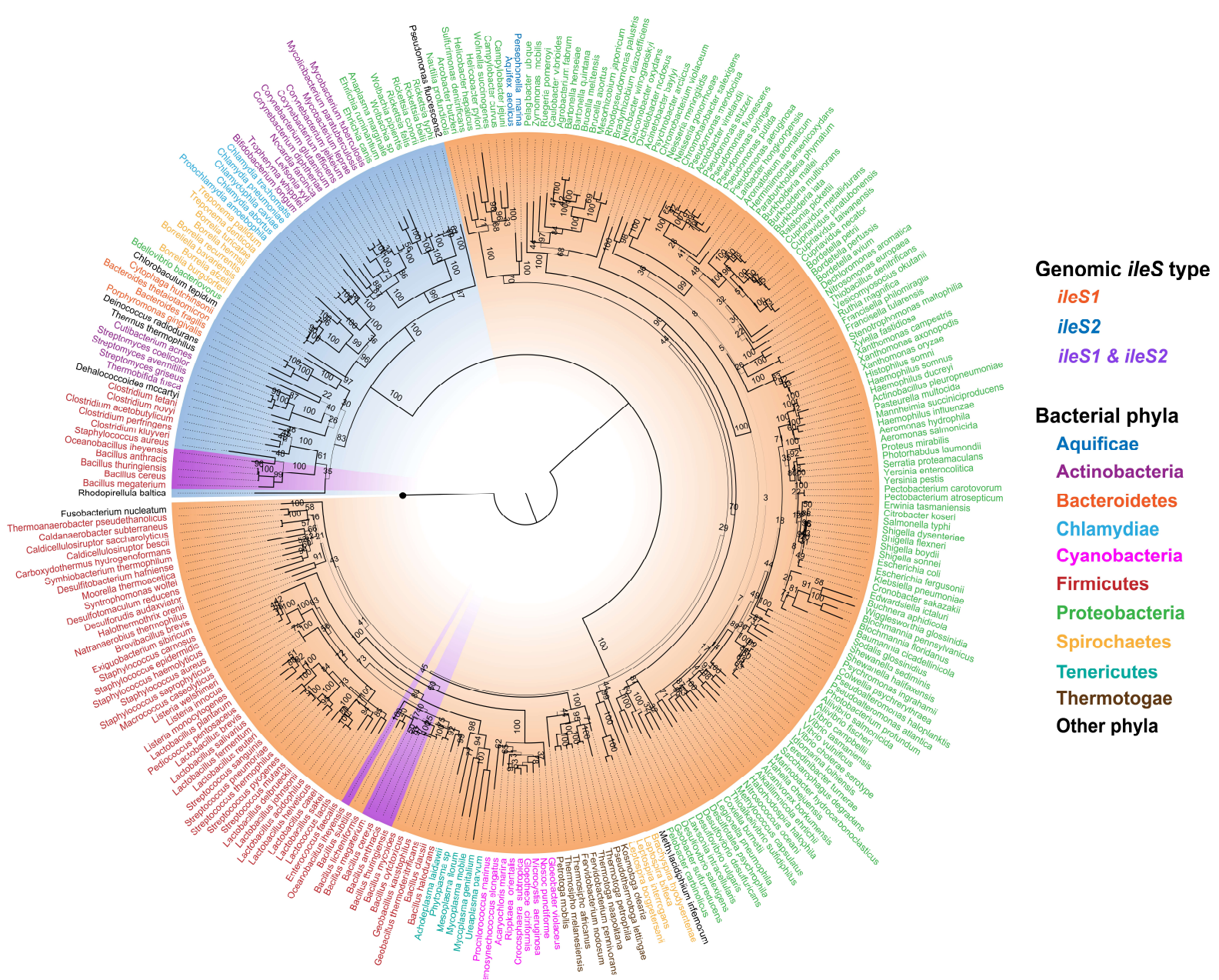

Figure 2. ileS distribution throughout bacterial kingdom.

Phylogenetic tree was constructed using maximum likelihood method RAxML after multiple sequence alignment of 278 bacterial IleRS (218 ileS1, 60 ileS2). The tree was rooted using $E$. coli ValRS (black circle). The line width of each branch is scaled according to the bootstrap support value, and numbers along the nodes represent the percentage of nodes reproducibility in 1000 bootstrap replicates. Species names are colored by phylum. Nodes are highlighted according to the genomic ileS distribution. The species having both ileS1 and ileS2 (highlighted purple) belong exclusively to the family Bacillaceae. Exception is Staphylococcus aureus harboring plasmid copy of ileS2. 

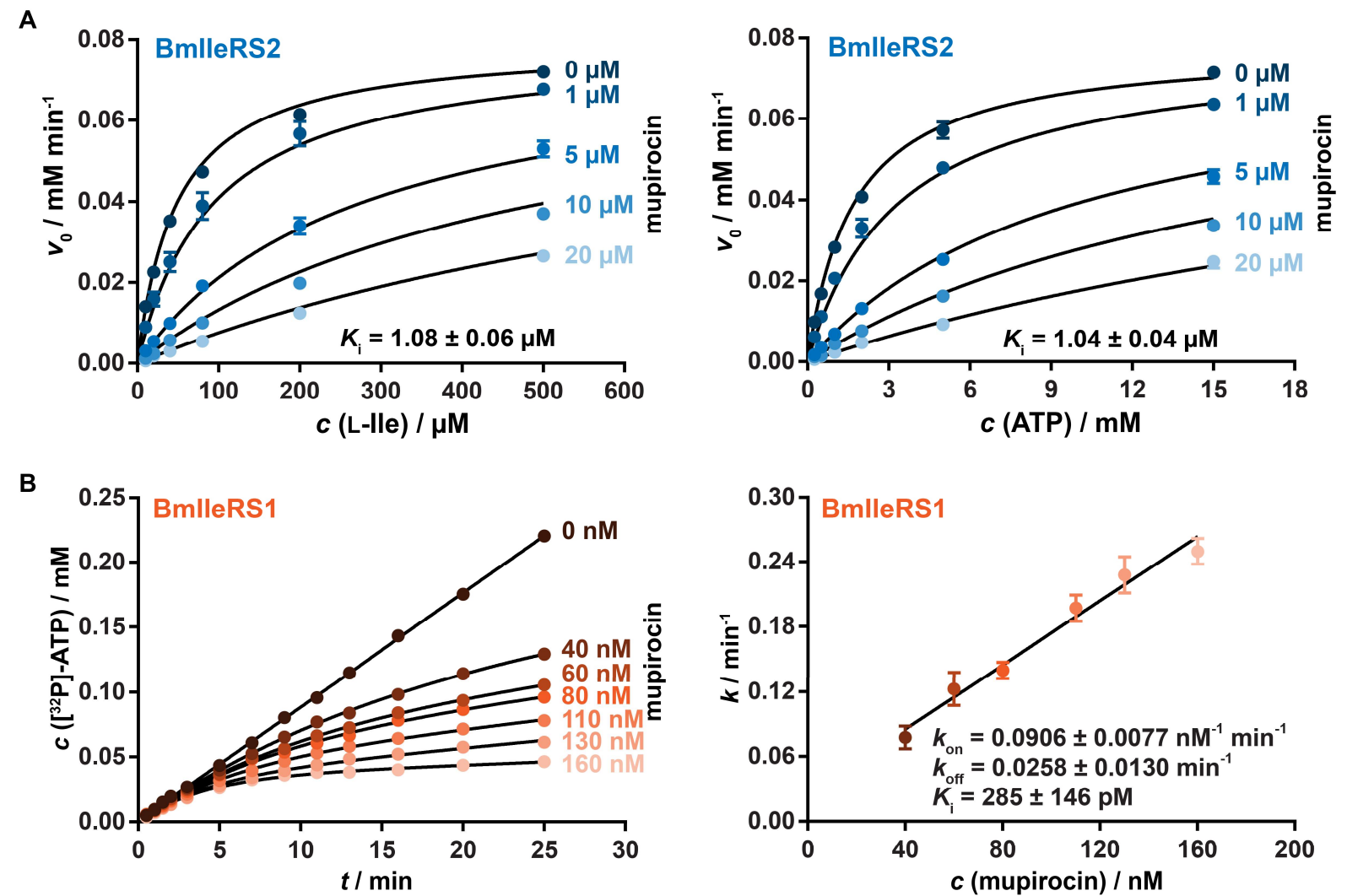

Figure 3. Inhibition of BmIleRS1 and BmIleRS2 by mupirocin.

Values represent the average value $\pm \operatorname{SEM}(n \geq 3)$.

A) BmIleRS2 displayed a classic fast-on/fast-off competitive inhibition in Ile activation. The figure depicts plots of initial reaction rates vs substrate concentration (left Ile, right ATP) in the presence of increasing concentrations of mupirocin. Data were fitted to classic competitive inhibition equation to obtain the inhibition constant $\left(K_{\mathrm{i}}\right)$.

B) BmIleRS1 displayed a slow, tight-binding competitive inhibition. The time-courses (left) were fitted to a slow-binding equation and the apparent rate constant $k$ was replotted vs mupirocin concentration to reach the $k_{\mathrm{on}}$ and $k_{\mathrm{off}}$ describing mupirocin binding. Inhibition constant $\left(K_{\mathrm{i}}\right)$ was calculated from the ratio of $k_{\text {off }}$ and $k_{\text {on }}$ (for details see Supplementary Materials and methods). 


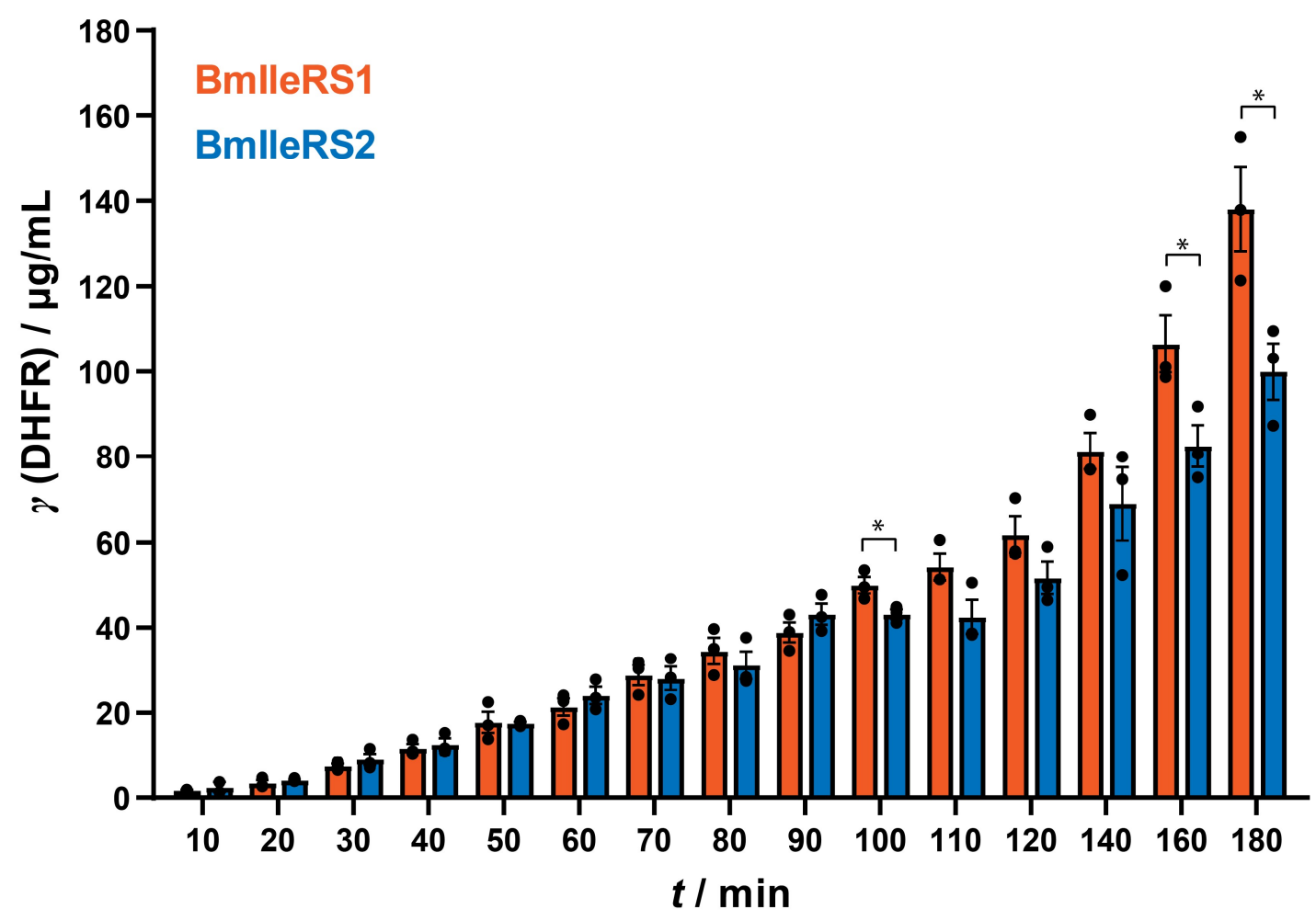

Figure 4. In vitro translation of dihydrofolate reductase using BmIleRSs.

The values represent the average value $\pm \operatorname{SEM}(n=3)$. Statistically significant difference in DHFR amount was calculated using Student's t-test and asterisk (*) indicates statistical difference with $\mathrm{p}<0.05$. 

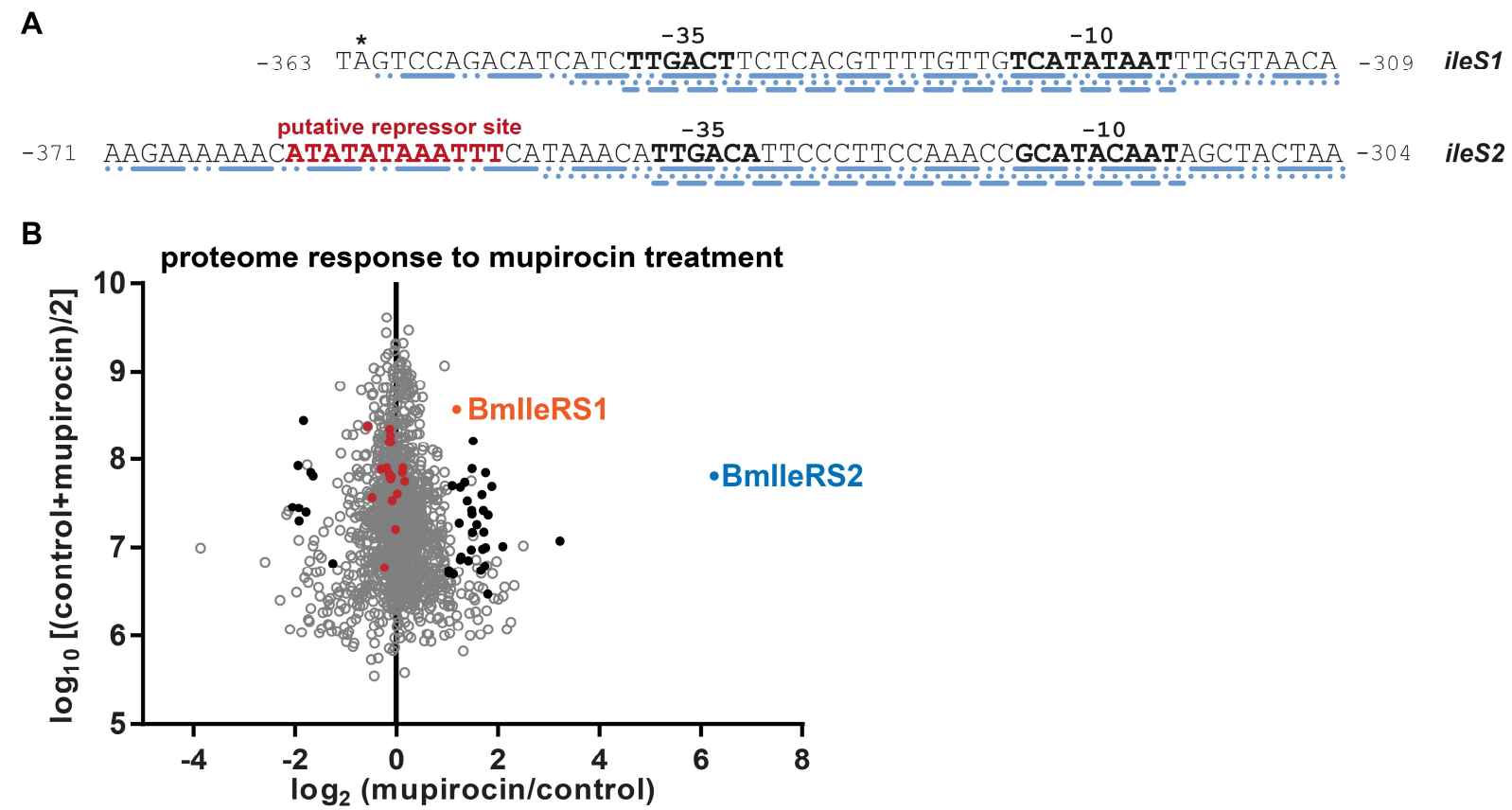

Figure 5. Putative transcriptional regulation and expression profiles of $B$. megaterium IleRSs.

A) Upstream region of ileS genes analyzed by BacPP $(\bullet-\bullet)$, PePPER $(--)$ and FruitFly $(\bullet)$. Numbering starts from the first nucleotide of the ORF (not shown). T-box regulatory sequences (not shown, Supplementary Figure S3A) are inserted between the first nucleotide of the ORF and a putative -10 promotor element; asterisk (*) denotes the stop codon of the upstream gene. SoftBerry analysis of $B$. megaterium putative -35 and -10 promoter elements (bold) indicate that rpoD16 and rpoD19 transcription factors (both part of $\sigma^{70}$ subunit) regulate ileS1 while lexA and $\operatorname{argR} 2$ transcription repressors (both involved in SOS response) regulate ileS2. A putative repressor binding site was independently localized based on multiple sequence alignment of Bacilli ileS2 (Supplementary Figure S3A).

B) Mass spectrometry proteome analysis revealed 70-fold upregulation of BmIleRS2 in the presence of mupirocin. Filled black circles represent proteins with more than 2-fold statistically significant change in expression including also BmIleRS1 (two sided, two sample Student's ttest with Benjamini-Hochberg correction for multiple hypothesis testing, FDR=0.05). The red circles mark other aaRSs. 
A

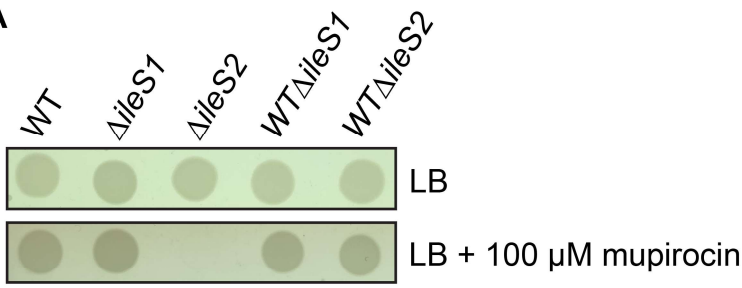

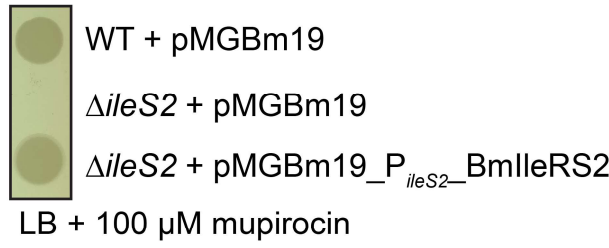

C

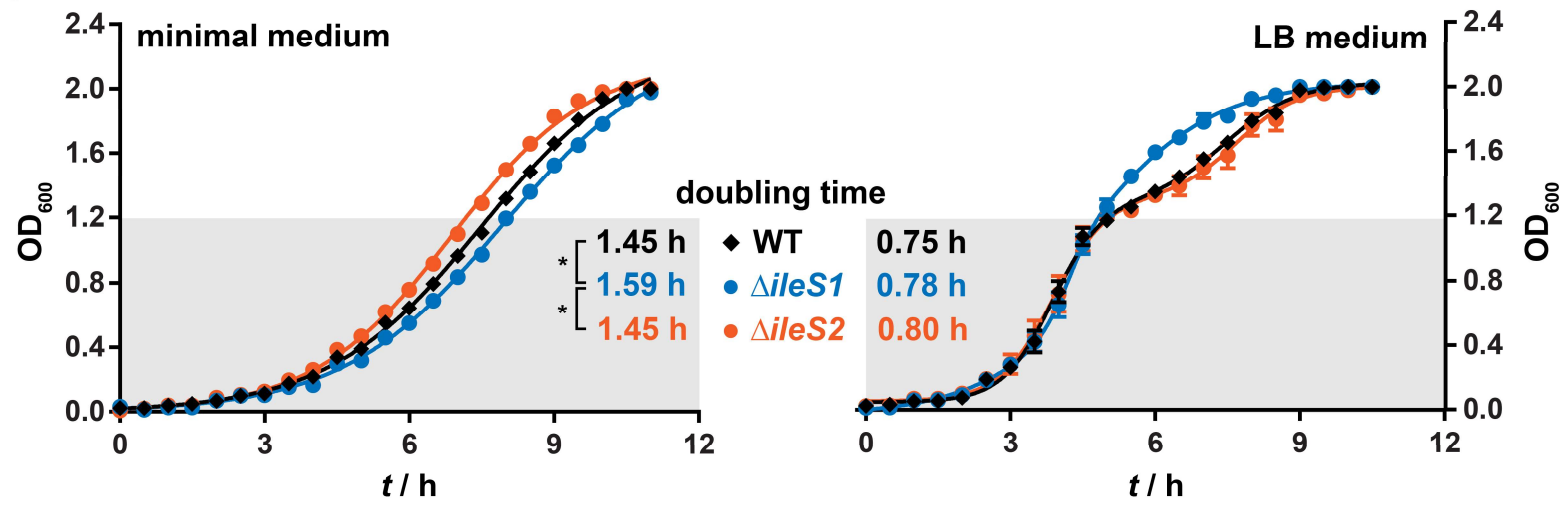

Figure 6. Phenotypic characterization of the B. megaterium knockout strains.

A) Both $\Delta i l e S 1$ and $\triangle i l e S 2$ were viable in the absence of mupirocin. $100 \mu \mathrm{M}$ mupirocin arrested growth of $\Delta i l e S 2$. WTAileS1 and WTAileS1 represent WT strain genotype after knockout procedure.

B) $\Delta i l e S 2$ mupirocin-sensitive phenotype was complemented by ileS2 expressed from its native promotor from the plasmid pMGBm19_P $\mathrm{P}_{\text {iles2_BmIleRS2. BmIleRS2 expression was }}$ confirmed by Western blot (Supplementary Figure S6A).

C) The growth curves were followed in minimal medium (left) and LB (right). The full profiles were fitted to logistic (left) and biphasic equations (right), the latter exemplified $\Delta i l e S 1$ unique phenotype in LB. The exponential phases $\left(\mathrm{OD}_{600}<1.2\right.$, marked with gray) were separately fitted to $y=y_{0} \times e^{r \times t}$ and the growth rates $(r)$ extrapolated. The calculated doubling time is depicted and the statistically significant differences ( $\mathrm{p}<0.05$ calculated by Student's t-test) are indicated with asterisks $\left(^{*}\right)(\mathrm{n} \geq 3)$. $\Delta i l e S 1$ exhibited an impaired growth rate only in minimal medium. 
A

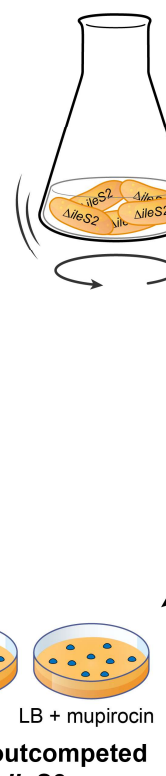

$\Delta$ iles2
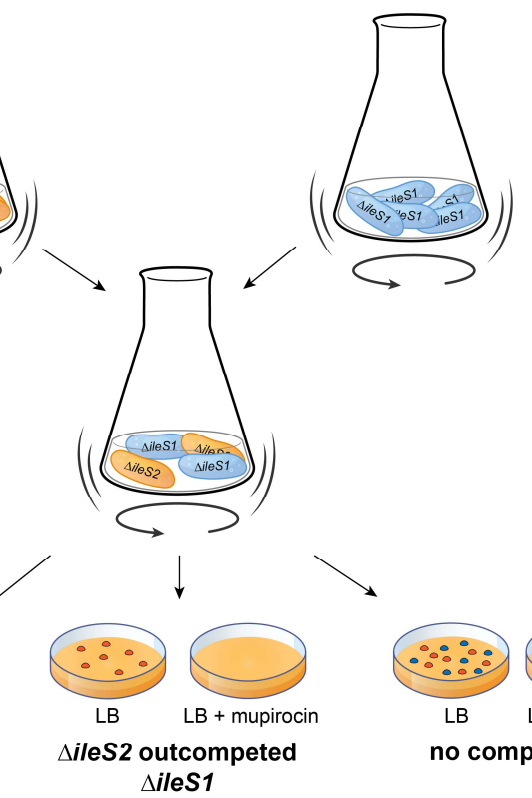

strains grown individually until mid-exponential phase

competition started by mixing equal amount of cells

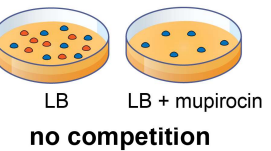

selection

$\Delta$ ileS2 cannot grow on mupirocin

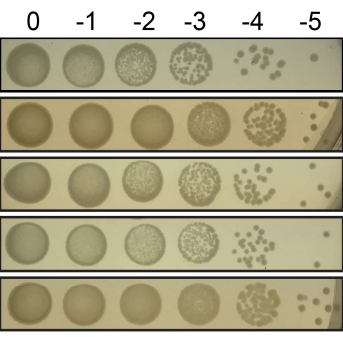

LB $\begin{array}{llllll}0 & -1 & -2 & -3 & -4 & -5\end{array}$

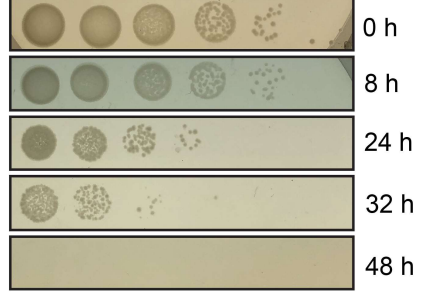

$\mathrm{LB}+1 \mu \mathrm{M}$ mupirocin

competition in minimal medium

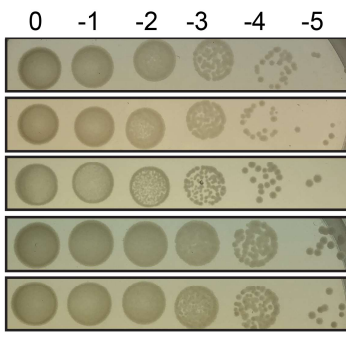

LB

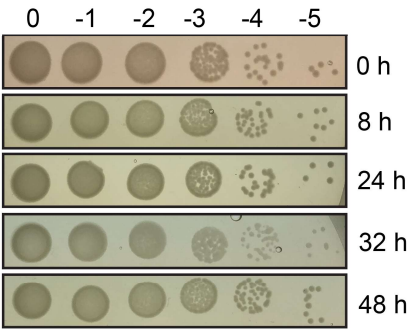

$\mathrm{LB}+1 \mu \mathrm{M}$ mupirocin

Figure 7. Competition between $\Delta i l e S 1$ and $\triangle i l e S 2$ strains.

A) Schematic presentation of the competition experiment.

B) Selection plates for the competition experiments performed in minimal medium (left) and LB medium (right) for indicated time (up to 48 hours). One representative experiment is shown. 
A

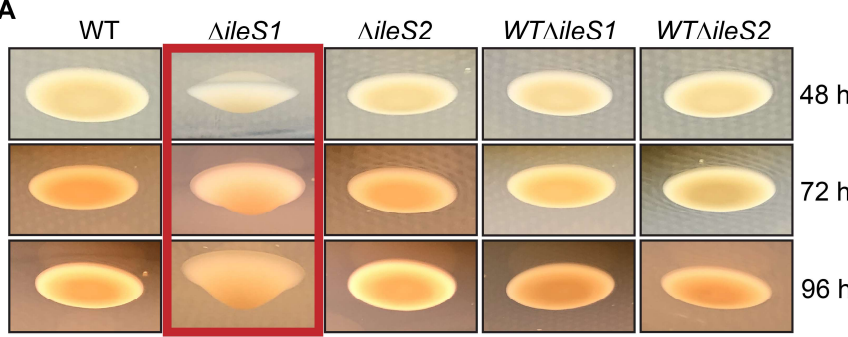

LBGM

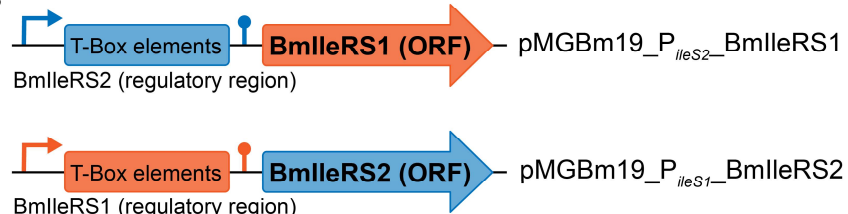

Figure 8. Time-dependent biofilm formation.

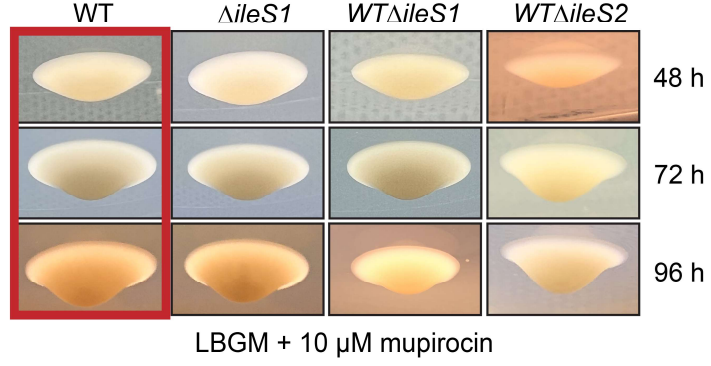

C

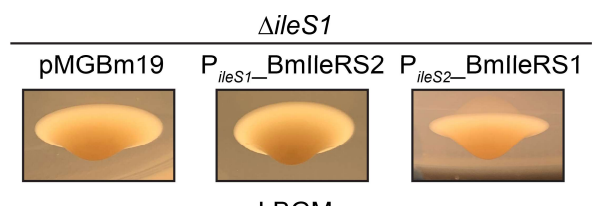

LBGM

The result of one representative experiment is shown.

A) The strains were grown in LB, spotted on the plates with biofilm promoting medium (LBGM) and incubated for up to 5 days at $30^{\circ} \mathrm{C}$. A "leaky" phenotype (marked as red squares) was observed in the absence of the functional IleRS1 (AileS1 strain or the WT strain, the latter only in the presence of mupirocin). WTAileS1 and WTAileS1 represent WT strain genotype after knockout procedure.

B) Schematic representation of the expression constructs having swapped regulatory (both promoter and T-Box riboswitch region) and ileS coding regions.

C) The AileS1 "leaky" phenotype could not be complemented by BmIleRS2 under ileS1 promotor or BmIleRS1 under ileS2 promotor. Expression of both enzymes from swapped promoters was confirmed by Western blot analysis (Supplementary Figure S6B). 


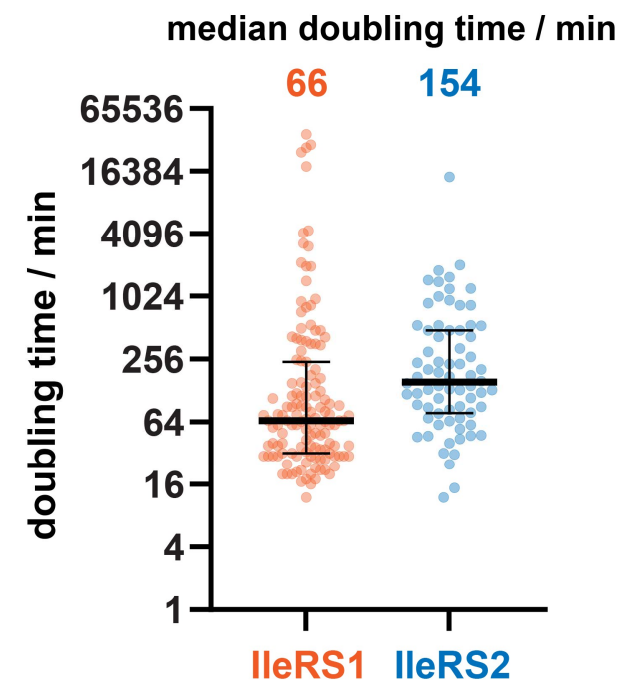

Figure 9. Relationship between IleRS type and bacterial doubling time.

Each dot represents one bacterial species colored according to the genomic IleRS type (n $(\mathrm{IleRS} 1)=135, \mathrm{n}(\mathrm{IleRS} 2)=72)$. Doubling times were compiled from the literature (if multiple conditions were reported the minimal doubling time was selected) and the median doubling times calculated. 